\title{
From Trigonometry to Number Theory... and Back: Extending LCM to Rational Numbers
}

\author{
Rina Zazkis • Jeffrey Truman
}

Published online: 24 March 2015

(C) Springer International Publishing 2015

\begin{abstract}
In order to explore the sum of sinusoids we extend the notion of least common multiple to rational numbers.
\end{abstract}

Keywords Sinusoid $\cdot$ sine $\cdot$ least common multiple $\cdot$ trigonometry $\cdot$ number theory

\section{Strange Questions}

\section{Is $1 / 2$ a factor of 4 ?}

What is the GCF (greatest common factor) of $1 / 2$ and $2 / 3$ ?

What is the LCM (least common multiple) of $1 / 3$ and $3 / 2$ ?

These questions make no sense, at least initially, as the notions of number theory, such as factor/divisor, multiple, GCF and LCM, are defined for natural numbers only. Actually, the mathematical resources are inconsistent in the scope of applicability of these notions, referring either to whole numbers, or natural numbers, or integers. However, rational numbers are definitely outside of the scope of conventional definitions for basic concepts in number theory. In this paper we extend some basic number theoretic notions to rational numbers and discuss the applicability of such extensions. We start with the motivation for the extensions, which relates to investigation of trigonometric functions, in particular the sum of sinusoids.

While the shape of a sinusoid is well known, to consider the sum of sinusoids without graphing technology is cumbersome. So we turn to the Geometer's Sketchpad (Jackiw 1988) to facilitate our exploration.

\section{Exploring Trigonometric Functions and Their Sums}

Consider the following function:

$$
f(x)=\sin x+\sin 2 x
$$

It is evident from the graph (Fig. 1) that the period of $\mathrm{f}(\mathrm{x})$ is $2 \pi$.

R. Zazkis $(\bowtie) \cdot J$. Truman

Simon Fraser University, Vancouver, BC, Canada

e-mail: zazkis@sfu.ca 


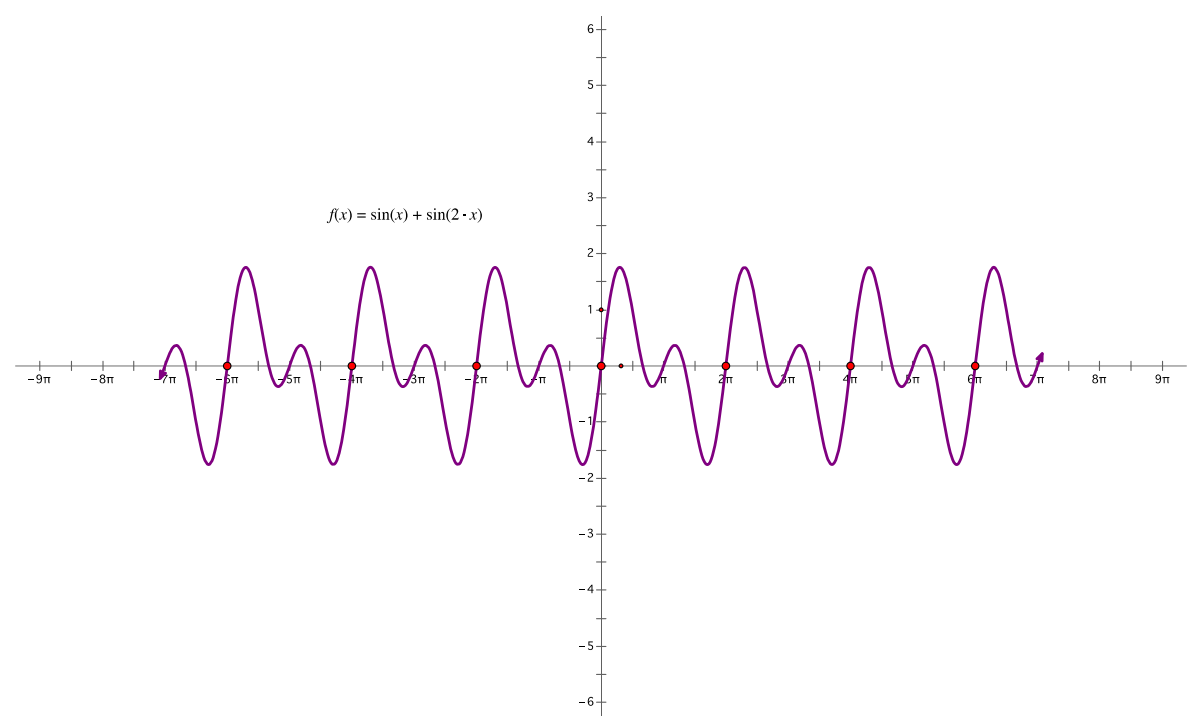

Fig. 1 Graph of $\sin (x)+\sin (2 x)$

This makes sense as the period of $\sin 2 \mathrm{x}$ is $\pi$, so for each wave of $\sin \mathrm{x}$ we get 2 waves of $\sin 2 x$ (Fig. 2). As such, the smaller period is subsumed by the larger.

Now consider $\mathrm{g}(\mathrm{x})=\sin 3 \mathrm{x}+\sin 5 \mathrm{x}$ (Fig. 3 ).

It is evident from the graph that the period of $\mathrm{g}(\mathrm{x})$ is also $2 \pi$ :

But why is this so?

A possible explanation is that the period of $\sin 3 x$ is $2 \pi / 3$, so it produces 3 waves within $2 \pi$, the period of $\sin 5 x$ is $2 \pi / 5$, so it produces 5 waves within $2 \pi$. As $2 \pi$ is the point where the end points of the iterations coincide, it is the period of the combined wave.

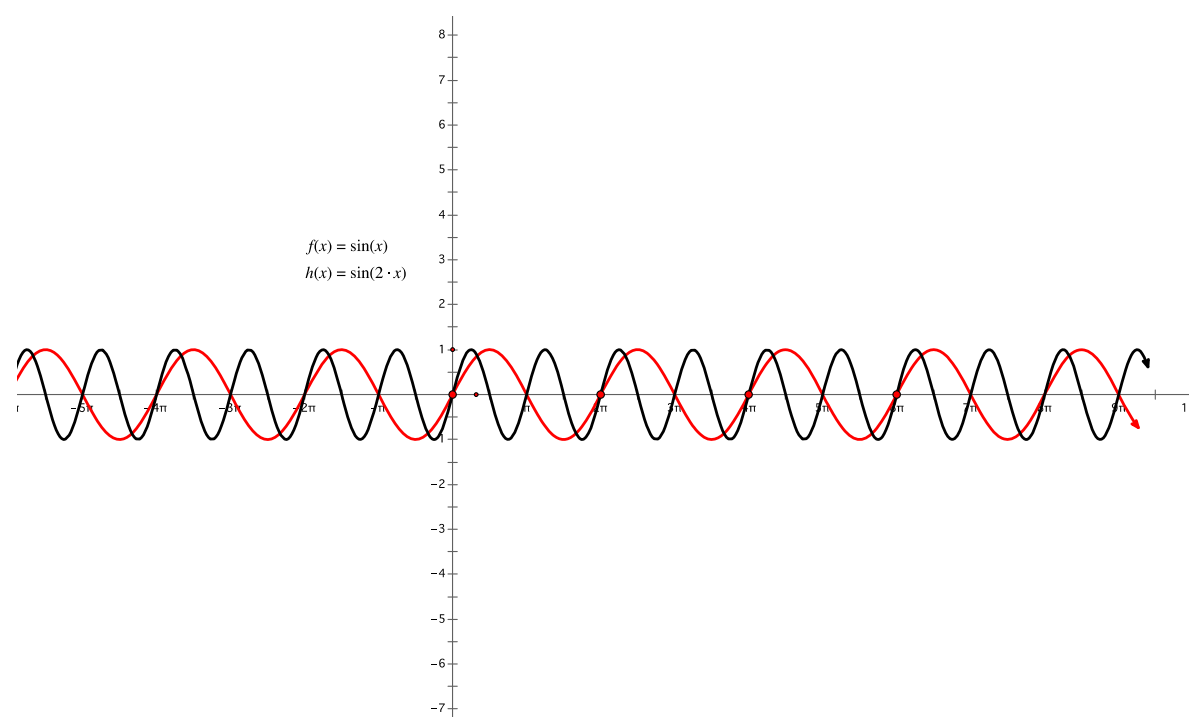

Fig. 2 Graphs of $\sin (x)$ and $\sin (2 x)$ 


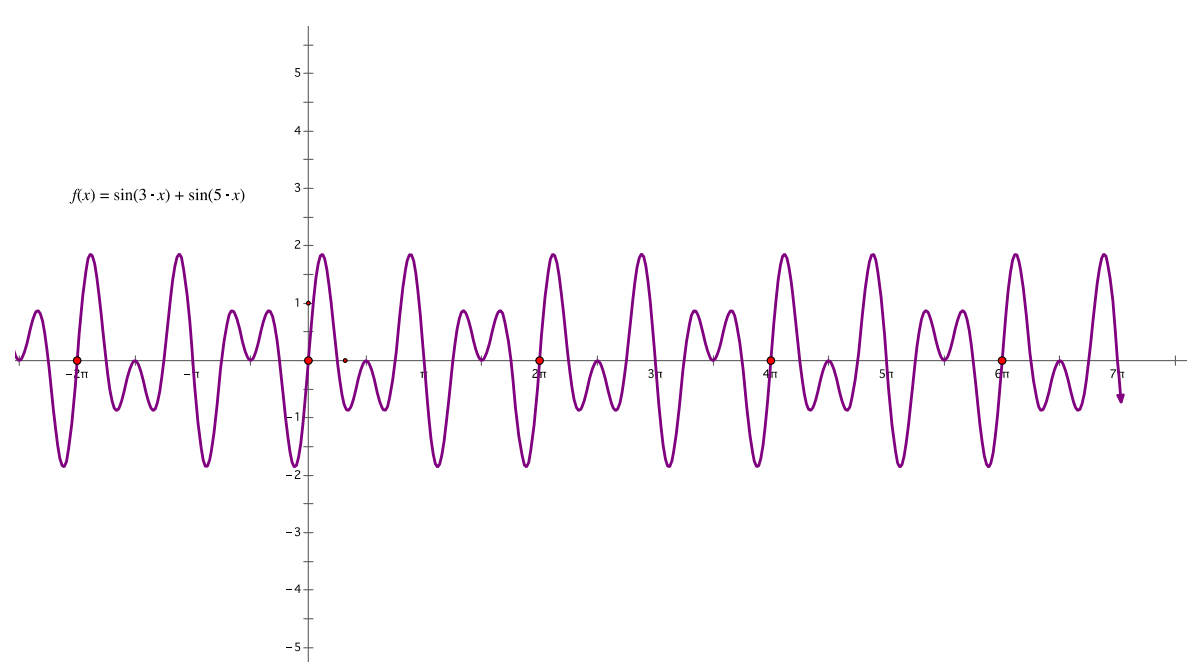

Fig. 3 Graph of $\sin (3 x)+\sin (5 x)$

However, working with numbers that are "less friendly", the decision is not immediate. Consider the sum of $\sin 8 x / 3$ and $\sin 16 x / 9$ (Fig. 4), or the sum of $\sin (20 x / 7)$ and $\sin (15 \mathrm{x} / 7)$ (Fig. 5).

It is hard to determine the exact value of the period of the sum function from the graph. However, the periods of individual addends are known. This brings us to the following question: Given that the period of one function is $\mathrm{a} / \mathrm{b}$ and the period of another is $\mathrm{c} / \mathrm{d}$, what is the period of their sum?

\section{On the Sum of Sinusoids}

The sum of sinusoids is usually discussed in the context of physics, as a combination of sound waves. In particular, the phenomenon of beats is often discussed in this context,

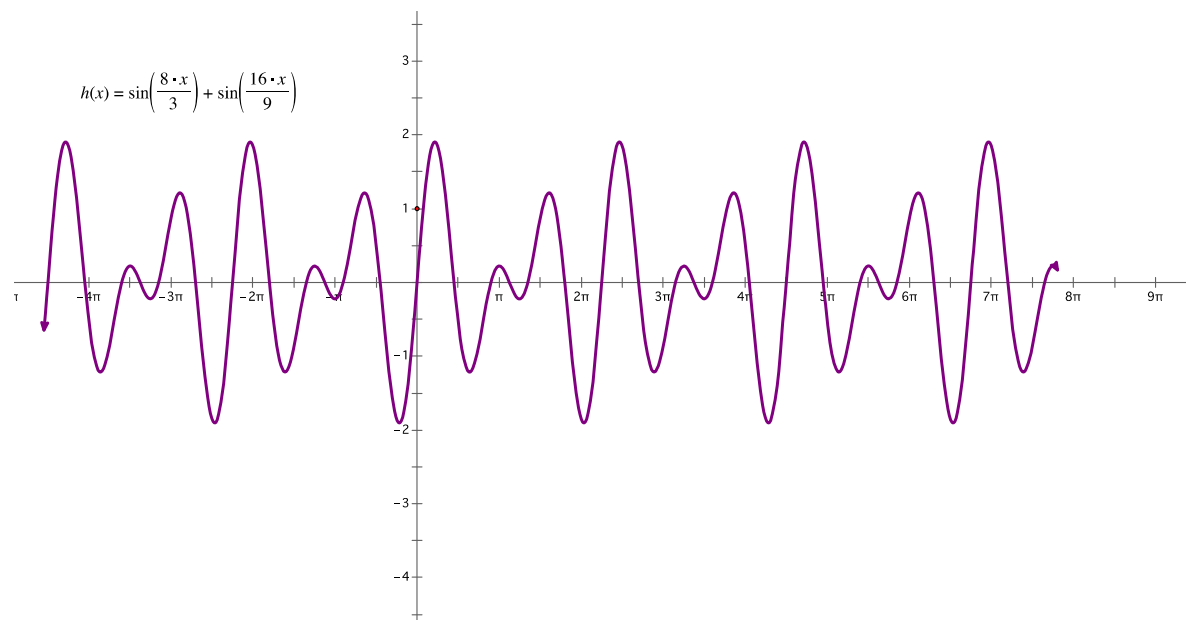

Fig. 4 Graph of $\sin (8 x / 3)+\sin (16 x / 9)$ 


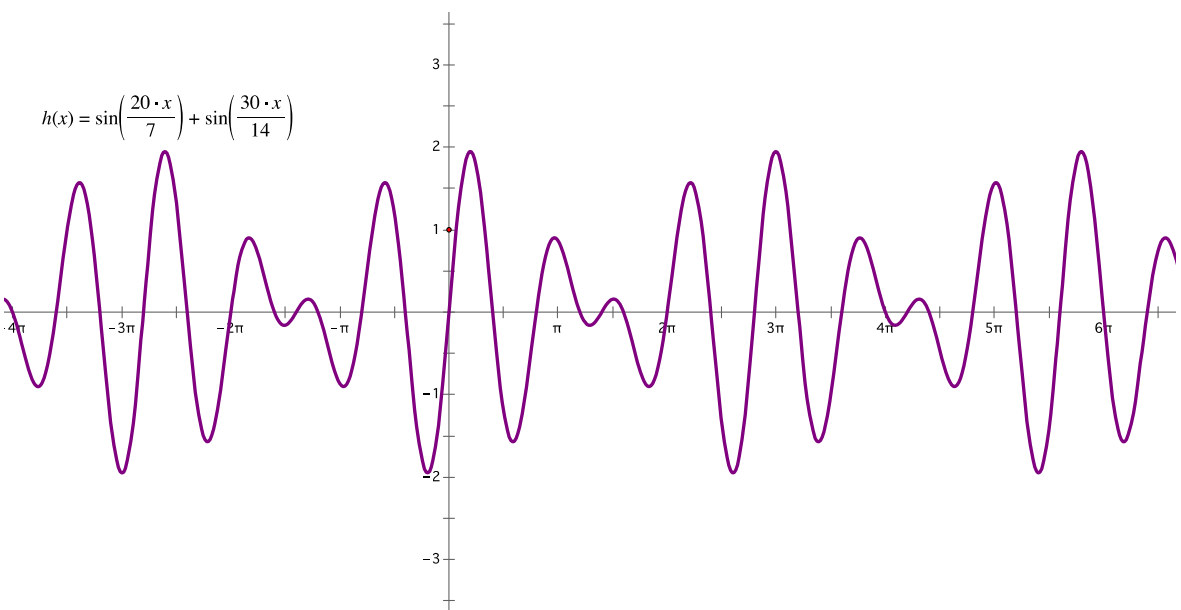

Fig. 5 Graph of $\sin (20 x / 7)+\sin (30 x / 14)$

sometimes in terms of wave packets and packet length. The frequency of the sum wave is given here as the difference of the two frequencies. However, it is noted that this physical phenomenon is discussed in relation to frequencies which are only slightly different. Here, we note that "slightly different" is generally either not defined, or imprecisely defined (such as a $20-30 \mathrm{~Hz}$ range). Through the extension of number theory principles we discuss, we can give a general method for finding packet length in the cases where such a phenomenon exists.

\section{Number Theory Connection}

Let us return to the sum of $\sin 3 x$ (function period is $2 \pi / 3$ ) and $\sin 5 x$ (function period is $2 \pi / 5$ ). Taking $\pi$ out of the equation, we imagine each period as a "jump" on a number line. If one proceeds with jumps of $2 / 3$, and another with jumps of $2 / 5$, where do they "land" on the same point? (Fig. 6) This image is similar to that of the least common multiple: the described landing points of "jumps" are multiples of different numbers, and the LCM is the first common landing point. The same number line image with a different labeling of points may serve for illustrating the common multiples of 3 and 5, for example (Fig. 7).

With this analogy in mind, we define a multiple-x (multiple-extended) of a rational number $\mathrm{a} / \mathrm{b}$ as follows:

A rational number $\mathrm{m} / \mathrm{n}$ is a multiple- $\mathrm{x}$ of a rational number $\mathrm{a} / \mathrm{b}$ if there exists a natural number $\mathrm{k}$ such that $\mathrm{a} / \mathrm{b} \times \mathrm{k}=\mathrm{m} / \mathrm{n}$. (This is analogous to the definition of $\mathrm{a}$ multiple for natural numbers $a, b$, and $k: b$ is a multiple of a if there exists $k$ such that $\mathrm{b}=\mathrm{a} \times \mathrm{k}$.)

Similar to the definition of LCM, we define the LCM-X (LCM - Extended) of two rational numbers as the smallest rational number which is a multiple of both.

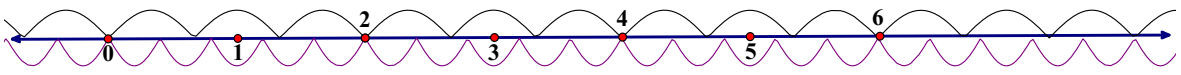

Fig. 6 Visual representation of $\operatorname{LCM}-\mathrm{X}(2 / 3,2 / 5)$ 


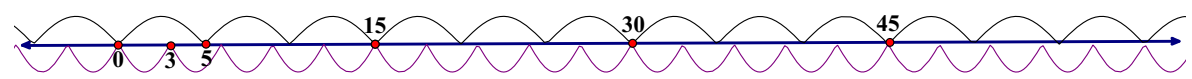

Fig. 7 Visual representation of $\operatorname{LCM}(3,5)$

So to determine the period of the sum of two sinusoids of the type $\sin (q x)$, for rational numbers $\mathrm{q}$, is to find LCM-X of 2 numbers, $\mathrm{a} / \mathrm{b}$ and $\mathrm{c} / \mathrm{d}$, which represent the periods of the two sinusoids.

We claim that $\operatorname{LCM}-\mathrm{X}(\mathrm{a} / \mathrm{b}, \mathrm{c} / \mathrm{d})=\operatorname{LCM}(\mathrm{a}, \mathrm{c}) / \operatorname{GCF}(\mathrm{b}, \mathrm{d})$. [A proof is presented in the Appendix.]

The issue of commensurability of rational numbers is usually mentioned only in the discussion of incommensurability of irrationals. It is claimed that any two rational numbers are commensurable, that is, there exists a unit that measures both. That is, the same unit "fits in" each of the numbers an integer number of times. However, what is the largest unit that measures both? For natural numbers, such "unit" is their GCF. Similarly, the notion of GCF can be extended to rational numbers. We define the GCF$\mathrm{X}$ of two rational numbers as the largest rational number that measures both.

We claim that GCF-X (a/b, c/d) = GCF $(a, c) / \operatorname{LCM}(b, d)$.

Commensurability of rational numbers assures the existence of LCM-X and GCF-X. However, a reasonable objection is expected: The periods of trigonometric functions (e.g., $2 \pi$ ) are not rational numbers. The formal definition of commensurability helps resolve the issue, since commensurability is not limited to rational numbers: Two real numbers $\mathrm{a}$ and $\mathrm{b}$ are commensurable if $\mathrm{a} / \mathrm{b}$ is a rational number. When periods of functions are expressed as $\mathrm{q} \pi$ for a rational $\mathrm{q}$, they are commensurable.

\section{Returning to Trigonometry: Examples}

The period of $\sin (8 x / 3)+\sin (16 x / 9)$ is $\pi \times \operatorname{LCM}-X(3 / 4,9 / 8)=(9 / 4) \pi$ which is shown in Fig. 8.

The period of $\sin (20 x / 7)+\sin (15 x / 7)$ is $\pi \times \operatorname{LCM}-X(7 / 10,14 / 15)=(14 / 5) \pi$, which is shown in Fig. 9.

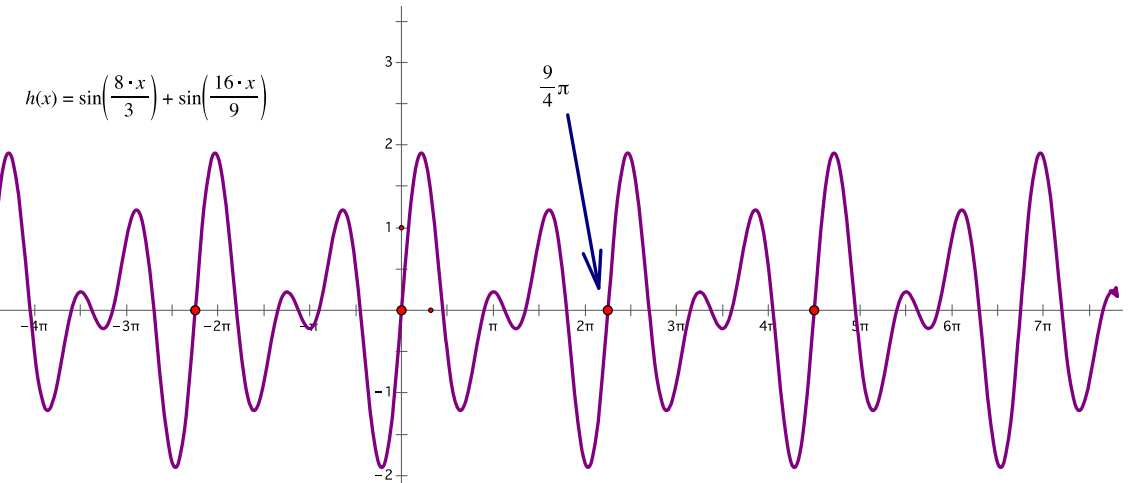

Fig. 8 The period of $h(x)=\sin (8 x / 3)+\sin (16 x / 9)$ is $(9 / 4) \pi$ 


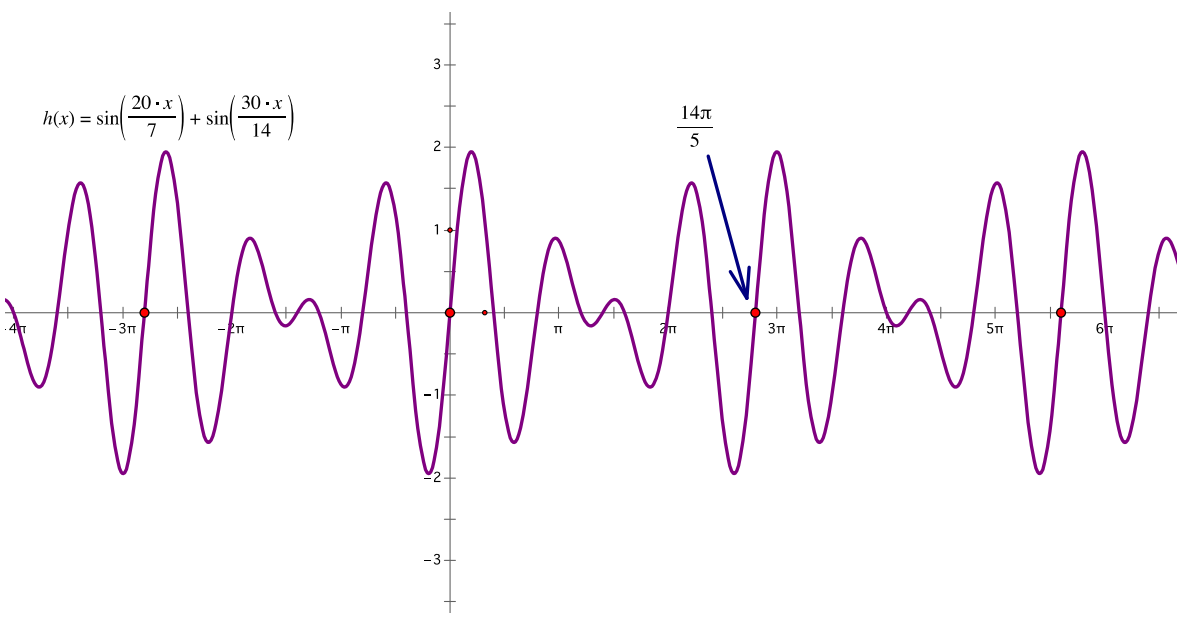

Fig. 9 The period of $h(x)=\sin (20 x / 7)+\sin (30 x / 14)$ is $(14 / 5) \pi$

\section{On Extending the Scope}

"Challenging basic assumptions" and extending the scope of applicability of conventional notions is a powerful mathematical activity (Zazkis 2008). It extends and strengthens the existing schemas for familiar notions. For example, experiencing computation in bases other than ten helps understand the conventional number system and gain further appreciation of the notion of place value. Experiencing an affine coordinate system helps reexamine familiar algorithms (such as finding a line parallel to the given line through a given point, or a distance between two given points) in the conventional Cartesian coordinate system and consider invariants.

In a similar fashion we suggest that examining number-theoretic concepts with rational numbers helps in gaining richer appreciation of these notions. And in addition to the pure theoretical investigation, there is a practical gain: ability to determine the period of the sum of periodic functions, when the periods are commensurable.

The ease of generating graphs is inseparable from this investigation. While we focused on the sum of sinusoids, the results are applicable - and can be verified - for any periodic functions. Thanks to the plot-function tool of the Geometer's Sketchpad our claims are verifiable with ease, and - we believe - the readers are eager to generate a few examples of their own.

\section{For the Classroom}

Questions regarding the sum of periodic functions provide a valuable avenue for students' engagement, particularly with applications such as physical models of waves, since they often involve such sums. Starting with the sum of two sinusoids, investigation can be extended to sums and differences of multiple periodic functions. A composition of periodic functions provides an interesting extension of the activity in a more purely mathematical sense. 
The digital environment assures immediate visualization and essential feedback that support the exploration. It also provides unlimited example space for testing and generating conjectures. However, the aforementioned advantages are common to a variety of digital applications. In our case, technology provides not only some experience with using the new ideas of LCM-X and GCF-X, but also a visual representation that gives the students a way to think about what those ideas mean in a sense other than the given definition. The digital environment also provides an avenue for bridging separate areas of mathematics, which do not usually overlap in "standard" curricula.

\section{Appendix}

Claim:

For rational numbers $\operatorname{LCM}-X(\mathrm{a} / \mathrm{b}, \mathrm{c} / \mathrm{d})=\operatorname{LCM}(\mathrm{a}, \mathrm{c}) / \operatorname{GCF}(\mathrm{b}, \mathrm{d})$.

Proof:

We first prove that $\frac{\operatorname{LCM}(\mathrm{a}, \mathrm{c})}{\mathrm{GCF}(\mathrm{b}, \mathrm{d})}$ is a multiple-x of $\frac{a}{b}$

Consider

$$
\frac{\mathrm{LCM}(\mathrm{a}, \mathrm{c})}{\mathrm{GCF}(\mathrm{b}, \mathrm{d})} \div \frac{a}{b}=\frac{\operatorname{LCM}(\mathrm{a}, \mathrm{c})}{\mathrm{GCF}(\mathrm{b}, \mathrm{d})} \times \frac{b}{a}=\frac{\operatorname{LCM}(\mathrm{a}, \mathrm{c})}{\mathrm{a}} \times \frac{b}{\mathrm{GCF}(\mathrm{b}, \mathrm{d})}
$$

It is a natural number, as a product of two natural numbers.

The conclusion that $\frac{\operatorname{LCM}(\mathrm{a}, \mathrm{c})}{\mathrm{a}}$ and $\frac{b}{\mathrm{GCF}(\mathrm{b}, \mathrm{d})}$ are natural numbers is based on the consideration of prime factorizations of numerators vs. denominators.

In a similar way, $\frac{\operatorname{LCM}(\mathrm{a}, \mathrm{c})}{\operatorname{GCF}(\mathrm{b}, \mathrm{d})}$ is a multiple-x of $\frac{c}{d}$, so it is a common multiple-x of $\frac{c}{d}$ and $\frac{a}{b}$.

We now prove that $\frac{\operatorname{LCM}(\mathrm{a}, \mathrm{c})}{\operatorname{GCF}(\mathrm{b}, \mathrm{d})}$ is the least common multiple-x of $\frac{c}{d}$ and $\frac{a}{b}$.

Clearly, $\operatorname{LCM}(\mathrm{a}, \mathrm{c})$ is a multiple-x of $\frac{c}{d}$ and $\frac{a}{b}$.

Any other a multiple-x of $\frac{c}{d}$ and $\frac{a}{b}$ is of the form $\frac{\operatorname{LCM}(\mathrm{a}, \mathrm{c})}{\mathrm{m}}$ for some rational number $\mathrm{m}$.

Let us assume, by contradiction, that there exists a rational number $\mathrm{m}$ such that

(*) $\frac{\operatorname{LCM}(\mathrm{a}, \mathrm{c})}{\mathrm{m}}$ is a common multiple-x of $\frac{c}{d}$ and $\frac{a}{b}$

and that

$(* *) \frac{\operatorname{LCM}(\mathrm{a}, \mathrm{c})}{\mathrm{m}}$ is smaller than $\frac{\operatorname{LCM}(\mathrm{a}, \mathrm{c})}{\mathrm{GCF}(\mathrm{b}, \mathrm{d})}$.

If $\mathrm{m}<\mathrm{GCF}(\mathrm{b}, \mathrm{d})$ then $(* *)$ does not hold.

If $m>\operatorname{GCF}(b, d)$ than it must have a prime factor different from either factors of $b$ or factors of $\mathrm{d}$.

In this case $\frac{\operatorname{LCM}(\mathrm{a}, \mathrm{c})}{\mathrm{m}}$ is not a multiple-x of $\frac{a}{b}$ or of $\frac{c}{d}$; so $\left(^{*}\right)$ does not hold.

Therefore $\mathrm{m}=\mathrm{GCF}(\mathrm{b}, \mathrm{d})$.

QED. 


\section{References}

Jackiw, N. (1988). The geometer's sketchpad. Emeryville: Key Curriculum Press.

Zazkis, R. (2008). Examples as tools in mathematics teacher education. In D. Tirosh (Ed.), Tools in mathematics teacher education. (in Handbook for Mathematics Teacher Education, Vol. 2, pp. 135156). Rotterdam: Sense publishers. 